\title{
Pesquisas com famílias de portadores de transtorno mental
}

\author{
Research with families of people with mental disorder \\ Investigación con familias de personas con trastorno mental
}

\begin{abstract}
Sueli Aparecida Frari Galera', Ana Carolina Guidorizzi Zanetti', Gabriela Cristina da Silva Ferreira", Bianca Cristina Ciccone Giacon'"', Lucilene Cardoso'

' Universidade de São Paulo, Escola de Enfermagem de Ribeirão Preto, Departamento de Enfermagem Psiquiátrica e Ciências Humanas. Ribeirão Preto-SP, Brasil.

"Universidade de São Paulo, Hospital das Clínicas da Faculdade de Medicina de Ribeirão Preto. Ribeirão Preto-SP, Brasil.

III Universidade de São Paulo, Escola de Enfermagem de Ribeirão Preto,

Departamento de Enfermagem Psiquiátrica e Ciências Humanas. (Doutoranda) Ribeirão Preto-SP, Brasil.
\end{abstract}

Submissão: 25/02/2010 Aprovação: 25/10/2010

\section{RESUMO}

A finalidade deste trabalho é apresentar uma reflexão crítica sobre os conhecimentos produzidos acerca dos temas: o impacto do transtorno mental na família, o impacto do ambiente familiar na evolução dos transtornos e a experiência da família com o adoecimento mental. As medidas de sobrecarga e de emoção expressa são úteis para orientar políticas públicas e a prática profissional, porém não nos dão um quadro multidimensional da experiência do cuidado. Pesquisas sobre a experiência familiar contribuem para a compreensão do sofrimento presente nesta experiência e das formas de enfrentamento adotadas pelas famílias, contribuindo também para percebermos as diferenças dos contextos culturais no enfrentamento das famílias.

Descritores: Enfermagem; Pesquisa; Transtorno mental; Relações familiares.

\section{ABSTRACT}

The purpose of this paper is to present a critical reflection on the knowledge produced on the topics: the impact of the mental illness in the family, the impact of the family environment in the evolution of the eating and the experience family with the mental illness. The measures of overload and express emotion are useful to guide public politics and the practice professional, however in they do not give a multidimensional picture to them of the experience of the care. Research on the familiar experience contribute to the understanding of the present suffering in this experience and of the forms of confrontation adopted by the families, also contributing to perceive the differences of the cultural contexts in the confrontation of the families. Key words: Nursing; Research; Mental disorders; Family relations.

\section{RESUMEN}

La finalidad de este trabajo es presentar una reflexión crítica de los conocimientos producidos acerca de los temas: el impacto del trastorno mental en la familia, el impacto en el ambiente familiar en la evolución de los trastornos y la experiencia de la familia con la enfermedad mental. Las medidas de sobrecarga y de emoción expresas son útiles para orientar políticas públicas y la práctica profesional, pero no nos da un cuadro multidimensional de la experiencia del cuidado. Investigaciones sobre la experiencia familiar contribuyen para la comprensión del sufrimiento presente en esta experiencia y de las formas de enfrentamiento adoptadas por las familias, contribuyendo también para que percibamos las diferencias de los contextos culturales en el enfrentamiento de las familias.

Palabras clave: Enfermería; Investigación; Trastornos mentales; Relaciones familiares. 


\section{INTRODUÇÃO}

Fortemente influenciada pela psicanálise e pela concepção moral da doença mental a psiquiatria teve a tendência de considerar a família como causadora da doença de seu familiar. Segundo estas perspectivas, a doença mental era resultado de interações familiares patogênicas ou de contextos familiares onde o aprendizado de valores morais era prejudicado ${ }^{(1)}$.

As mudanças na política de saúde e social, os avanços no tratamento farmacológico, a desinstitucionalização e a substituição do modelo focado na cura e centrado no hospital pelo modelo de reabilitação biopsicossocial ocorridas na década de 50, contribuíram para aumentar o número de pessoas com doença mental grave vivendo na comunidade ${ }^{(2-3)}$.

A diminuição da oferta de leitos hospitalares e a criação de uma rede de serviços na comunidade deslocaram a responsabilidade pelo seguimento e evolução do tratamento da doença mental das equipes hospitalares para as equipes comunitárias, o doente e seus familiares. Como resultados desse processo de mudança estão ocorrendo transformações no papel das instituições, dos profissionais, dos doentes e de suas famílias relacionados ao cuidado em saúde mental.

De causadora da doença mental de seus membros, a família passou a ser considerada aliada nos cuidados com o adoecido. Neste sentido, as pesquisas passaram a considerar o impacto do transtorno mental na família, o impacto do ambiente familiar na evolução dos transtornos e a experiência família com o adoecimento mental. A finalidade deste trabaIho é apresentar uma reflexão sobre os conhecimentos produzidos acerca desses temas.

\section{IMPACTO DO TRANSTORNO MENTAL NA FAMÍLIA}

Este campo da pesquisa busca identificar os aspectos da vida familiar afetados quando a família tem entre seus membros um portador de transtorno mental. O resultado da convivência com o adoecimento mental é estudado principalmente através do conceito de sobrecarga, definido como "um estado psicológico que advém da combinação de trabalho físico, emocional e pressão social, assim como a restrição econômica que emerge do cuidar dos pacientes"(4).

Há, basicamente, dois tipos de sobrecarga, a objetiva e a subjetiva. A primeira refere-se às consequências negativas concretas que podem ser observadas e estão relacionadas com prejuízos em relação à rotina, vida social e profissional dos familiares, perdas financeiras, cuidados com o paciente, atenção a comportamentos inadequados, agressões físicas e verbais, condutas sexuais inapropriadas etc. $\mathrm{O}$ aspecto subjetivo da sobrecarga se refere a como o familiar cuidador se percebe e avalia estar nessa situação(5). Estudos mostram que os comportamentos do doente decorrentes de sintomas negativos da esquizofrenia são percebidos como os que causam maior sobrecarga objetiva, quando comparados aos comportamentos decorrentes de sintomas positivos. Este resultado é explicado porque os sintomas negativos incluem uma variedade de dificuldades funcionais e cognitivas temporariamente estáveis em relação aos positivos que são episódicos ${ }^{(6-7)}$.
Além disso, cuidadores que percebem o paciente como incapaz de alterar seus comportamentos decorrentes dos sintomas negativos e de cumprir certos papéis e obrigações, podem assumir responsabilidades extras, acarretando maiores níveis de sobrecarga objetiva ${ }^{(7)}$. Outro dado importante é que familiares de pacientes que tiveram mais de duas internações psiquiátricas apresentam maiores níveis de sobrecarga subjetiva, sugerindo o aumento do impacto com o tempo ${ }^{(8)}$.

A sensação de estresse e a sobrecarga percebida por familiares de portadores de esquizofrenia são semelhantes, independentemente se vivem ou não na mesma casa do doente. Os fatores que explicam a sobrecarga entre estes familiares são a preocupação com a doença do familiar e a tensão em seus próprios relacionamentos conjugais ${ }^{(9)}$.

Partindo da ideia de que o estado de sobrecarga varia de acordo com o estado do doente, um estudo alemão ${ }^{(10)}$ descreveu diferentes fases da sobrecarga familiar, observadas durante um ano após a alta hospitalar. São elas: nível de sobrecarga constantemente elevado devido à função psicossocial prejudicada; sobrecarga elevada devido à deterioração causada pelos sintomas da doença; redução contínua da carga após a recaída ou episódio recente; e substituição da carga.

Vários estudos indicam também que a satisfação com os sistemas de ajuda e apoio está associada com a diminuição de sobrecarga objetiva e subjetiva( ${ }^{(7)}$. Nesta perspectiva, a sobrecarga pode ser um indicador da qualidade da relação da família com o sistema de saúde que ela usufrui como suporte para cuidar de seu doente. Este aspecto é extremamente importante, pois permite avaliar o impacto dos serviços ofertados aos portadores de transtorno mental grave e persistente como a esquizofrenia.

Assim, com o aumento das pesquisas em saúde mental e a importância de seus resultados, o ônus que o adoecimento mental crônico impõe para a pessoa doente, seus familiares e para a sociedade colocaram a doença mental na agenda da saúde pública internacional ${ }^{(11)}$. A Organização Mundial da Saúde reconhece a sobrecarga familiar como um dos indicadores do impacto do transtorno mental no âmbito global ou internacional ${ }^{(12)}$.

Embora as pesquisas que investigam a sobrecarga familiar sejam frequentes na literatura internacional, e este possa parecer um tema esgotado, muitos autores defendem sua continuidade. Primeiro, porque, com as mudanças sociais, a sobrecarga pode se modificar ao longo do tempo; segundo, porque essas mudanças são indicadoras de necessidades e de resultado da assistência prestada no momento.

Outro aspecto importante, apontado na literatura, em defesa da manutenção de pesquisas que medem a sobrecarga familiar, é que ainda faltam estudos em países com diferentes níveis de desenvolvimento e pobreza. A aplicação de escalas para descrever a sobrecarga em diferentes países permitiria testar a generalidade do conceito, bem como verificar como ele se comporta em outros cenários onde as condições sociais e os relacionamentos familiares sejam diferentes ${ }^{(6,8)}$.

Um dos poucos estudos que avaliam a sobrecarga familiar em um país subdesenvolvido constatou que todos os cuidadores apresentaram um alto grau de sobrecarga, especialmente 
quando os cuidadores são mães, pessoas mais velhas, desempregados, quando apresentam baixo nível de escolaridade, e quando o paciente cuidado é mais jovem ${ }^{(13)}$. Estudo realizado no Brasil, com familiares de portadores de esquizofrenia, identificou três tipos de sobrecarga familiar: financeira, nas rotinas familiares e a sobrecarga em forma de doença física ou emocional. Foram observadas também alterações nas atividades de lazer e nas relações sociais da família ${ }^{(14)}$.

Inúmeros estudos internacionais utilizam instrumentos de medida validados para avaliação sistemática da sobrecarga familiar $^{(5)}$. Porém, no Brasil, há poucas publicações relatando este tipo de pesquisa e seus resultados. Um dos motivos da escassez de pesquisas sistemáticas nessa área pode ser a carência de instrumentos de medida validados e adaptados para o contexto brasileiro. Os autores citam somente dois trabalhos brasileiros que utilizaram escala de medida de sobrecarga validada, ambos dirigidos para cuidadores de pacientes idosos.

Como se pode observar, medidas da sobrecarga do cuidado são úteis em diversos aspectos da pesquisa e da prática profissional. No Brasil este tipo de pesquisa está se iniciando com a validação e aplicação de instrumentos confiáveis.

\section{O impacto do ambiente familiar na evolução do transtor- no mental}

Em algumas circunstâncias, o ambiente familiar pode contribuir negativamente com a evolução da doença provocando recaídas, as quais muitas vezes, exigem novas hospitalizações. Os principais estudos sobre como o ambiente familiar pode interferir na evolução da doença são aqueles relacionados ao conceito de Emoção Expressa (EE) ${ }^{(16)}$. Este conceito foi introduzido a partir de estudos que investigaram fatores relacionados às recaídas psiquiátricas de pacientes com esquizofrenia, após a alta hospitalar.

Emoção expressa é um conceito desenvolvido para estudar o ambiente familiar ou as relações familiares. É uma medida do número de emoção tipicamente exposta no ambiente familiar, no dia a dia nas relações familiares que envolvem o cuidado com o familiar mentalmente adoecido. Esse conceito trata da qualidade da interação social entre os membros da família(17).

A EE vem sendo utilizada como um índice que inclui as seguintes dimensões: o número de comentários críticos (avaliação negativa da conduta do paciente); hostilidade (avaliação negativa do paciente como pessoa) e o super envolvimento emocional (sentimentos ou atitudes, por parte dos familiares, de desesperança, auto sacrifício, superproteção acerca do paciente). A família pode ser classificada com elevada $E E$, quando pelo menos um de seus membros apresenta uma ou mais dessas dimensões ${ }^{(18)}$. A maioria desses estudos foi desenvolvida em países de língua inglesa.

$\mathrm{Na}$ atualidade, há um crescente interesse em investigar o conceito de EE em diferentes contextos culturais. Um exemplo desta tentativa é um estudo realizado na China ${ }^{(19)}$, o qual mostrou a validade da EE na China. Esse autor aponta como diferença cultural, o nível de tolerância, entre os membros familiares acerca da pessoa com esquizofrenia, mostra que as percepções dos pacientes e os modos de lidar com os diferentes níveis de EE diferem culturalmente.
Nessa vertente, recomenda-se a aplicação das dimensões da EE em outros contextos culturais, para conhecer os dados normativos da população antes de associar a emoção expressa com as recaídas de doenças ${ }^{(20)}$. Há evidências que a evolução da esquizofrenia é melhor em países em desenvolvimento, atribuído à baixa emoção expressa nesses países.

No Brasil, após ampla revisão da literatura acerca dos instrumentos utilizados para avaliar a EE, identificou-se a versão abreviada da Entrevista Familiar de Camberwell - EFC como o único instrumento traduzido para língua portuguesa ${ }^{(21)}$. Porém, ao considerar que a EFC apresenta como limitações o tempo gasto e a necessidade de treinamento específico para a sua aplicação, ela é pouco utilizada. Deste modo, ainda existem poucos estudos que investigam o ambiente familiar do paciente com esquizofrenia no Brasil.

\section{A experiência de conviver com o adoecimento de um familiar}

A experiência de conviver com o adoecimento de um familiar é estudada por meio de pesquisas qualitativas fundamentadas em distintos referenciais teóricos e comportam diferentes metodologias.

Essas pesquisas têm produzido uma gama variada de informações e conhecimentos, que abrangem desde condutas, conflitos, emoções e sentimentos habituais entre os familiares, até práticas relativas ao cuidado. A coleta de dados geralmente se faz através de entrevistas aprofundadas com um ou mais familiares de doentes mentais, na maioria das vezes, portadores de esquizofrenia. As análises das entrevistas fundamentam-se nos referenciais adotados. Destacamos, a seguir, algumas dessas contribuições.

As famílias e, em especial, os pais vivem em um estado de angústia e preocupação constantes e sentem-se culpados pela doença de seus filhos e familiares. Enquanto este sentimento de culpa permanece silenciado a família não consegue aceitar que o familiar doente não é aquela pessoa independente, coerente que ela gostaria que fosse. Além disso, as questões associadas ao futuro, tais como quem vai ficar com o doente, trazem muitas preocupações para os pais e cuidadores, que esperam constantemente por uma vida melhor para o seu filho ${ }^{(3,22)}$

As narrativas dos pais revelaram também um conflito cultural entre o sistema familiar e o sistema do cuidado, o qual foi interpretado como uma ameaça ao papel parental, embora reconheçam que o sistema de cuidados oferece conforto e eles tenham confiança no cuidado dado ${ }^{(3)}$. Familiares estão sempre atentos para as alterações de comportamento de seu doente, evitam impor muitos limites para que ele não se irrite, procuram-se antecipar às suas necessidades e sentem-se frustrados, pois, apesar de toda sua dedicação não recebem muito afeto do doente ${ }^{(22)}$.

Outra pesquisa indicou que a principal tarefa da família é lidar com a permanência do filho doente no convívio dos pais. Para muitos pais altamente sobrecarregados, viver na mesma casa do indivíduo doente é extremamente exaustivo, pois a doença do filho é testemunhada diretamente e vivida intensamente. Os pais enfrentam também dificuldade moral e social ao se deparar com a possibilidade/necessidade de 
transferir o cuidado que o seu filho exige para uma instituição de saúde. Vivenciam frustrações pelo não cumprimento da expectativa de vida sonhada para o filho e pela diferença entre ele e outros membros da família( ${ }^{(23)}$.

Em relação às emoções vivenciadas pelos familiares, elas são caracterizadas por muita ansiedade quanto à maneira correta de lidar com o paciente e quanto aos problemas aumentados na vida familiar. Os familiares percebem seus doentes isolados e preocupam-se com isso, porém demonstram apatia, tristeza, desânimo e indecisão em suas ações ${ }^{(23)}$.

Em um estudo realizado com famílias de adultos jovens portadores de esquizofrenia, com o objetivo de interpretar as práticas de cuidados familiares que se desenvolvem em resposta ao surgimento da esquizofrenia, identificou quatro modelos distintos de práticas de cuidados: cuidado engajado, cuidado conflituoso, cuidado administrável e cuidado distanciado(24).

Um estudo comparou as respostas de familiares canadenses e japoneses, focalizando as mudanças na família após o diagnóstico de doença mental. A pesquisa, orientada pela teoria de enfermagem de Newman, descreveu dois tipos de padrões de relacionamento familiar nas famílias dos dois países: um quando o paciente está estável; e outro, quando o familiar doente está desorganizado. A autora verificou que os canadenses vivem os seguintes momentos: esforçam-se, resistem e sofrem sozinhos; transformam a si mesmos para incluir a doença mental no seu cotidiano. Diferentemente, os familiares japoneses vivem momentos em que não reconhecem a doença, porém resistem, sofrem e se transformam como família ${ }^{(25)}$.

Outro estudo focalizando a experiência de mães tailandesas de portadores de esquizofrenia verificou que elas buscam no budismo formas de conviver com seus filhos doentes. Praticam o Thum-jai com a finalidade de criar um ambiente calmo na família(26).

Os estudos brevemente descritos acima apontam para a existência de um processo de ajustamento familiar ao longo do tempo. Porém em todos eles os participantes da pesquisa conviviam com o adoecimento por diferentes períodos de tempo, dificultando estabelecer com maior clareza os momentos de ajustamento.

\section{CONCLUSÃO}

Com a mudança do ambiente de cuidado do familiar com transtorno mental, as famílias passaram a experimentar um novo ambiente cheio de emoções, responsabilidades e mudanças $^{(3)}$. Com o intuito de cuidar do doente neste novo ambiente, muitos estudos começaram a ser realizados, tendo como foco principal entender como as respostas familiares e do ambiente em que o doente se encontrava poderiam interferir no processo da doença. Duas áreas importantes de investigações podem ser destacadas, a sobrecarga e a emoção expressa.

Os estudos que investigam sobrecarga familiar indicam que os sintomas negativos da doença aumentam a sobrecarga objetiva da família; que não há diferença significativa entre a sobrecarga de famílias que vivem na mesma casa do doente e daqueles que moram em casas separadas; que quanto maior a gravidade dos sintomas, maior a sobrecarga; que quanto maior o número de internações, maior a sobrecarga subjetiva; e que a satisfação da família com o sistema de ajuda e apoio está relacionado diretamente com a diminuição da sobrecarga objetiva e subjetiva $^{(6-9)}$.

Têm sido desenvolvidos estudos para descrever a trajetória de vida de família e sua experiência perante o convívio com a doença. Eles mostram que a experiência da família pode sofrer alterações devido a aspectos como o aprendizado sobre a doença e as maneiras de enfrentamento que proporcionem uma melhor qualidade de vida ao grupo familiar. Isso pode interferir, por exemplo, no tipo de sobrecarga que a doença poderá causar na família.

As pesquisas com EE investigam a relação entre o elevado nível de EE com a frequência de recaídas e re-hospitalização. Estes estudos indicam que pacientes que recebem alta hospitalar para ambientes familiares com elevados níveis de estresse, resultam em exacerbação dos sintomas e ou em recaídas e que quando os familiares e cuidadores se comportam de maneira verbal negativa com os pacientes, pode produzir estresse, deixando-os vulneráveis a psicose e outras doenças ${ }^{(27)}$.

Porém, quando o conceito de EE é compreendido de maneira inadequada pelos profissionais de saúde, faz com que os estes vejam apenas a interação negativa da família com o doente, provocando conflito entre o serviço de saúde e as famílias e consequentemente, o distanciamento de ambos ${ }^{(28)}$.

A sobrecarga familiar e o ambiente familiar configuram importantes aspectos para a compreensão da relação entre o impacto da doença mental na família, e da família na evolução do transtorno mental. Entretanto, essas duas medidas focalizam apenas um aspecto da relação doença mental e família. Devemos nos lembrar de que a relação família e doença mental se constrói da junção: influência da doença na família e desta no percurso da doença e a experiência de ambos frente essa nova realidade.

Em relatos de familiares, pode-se observar frequentemente que referem sentir que os profissionais de saúde mostram pouco entendimento sobre como é para eles a experiência da doença mental, a sobrecarga e a imensa tristeza vivenciada. Por não conseguirem compreender a complexidade de como é viver com a doença mental, seus conselhos, muitas vezes, são vistos por essas famílias como simplistas e inapropriados ${ }^{(28)}$.

Assim, as medidas de sobrecarga e de emoção expressa são úteis para orientar políticas públicas e orientar a prática profissional, porém não nos dão um quadro multidimensional da experiência do cuidado. Neste sentido se destaca a relevância das pesquisas qualitativas sobre a experiência familiar ${ }^{(3,28)}$.

As pesquisas sobre a experiência familiar de conviver com o adoecimento mental contribuem para a compreensão do sofrimento presente nesta experiência e das formas de enfrentamento adotadas pelas famílias. Aliviando assim a culpa que as famílias, principalmente as mães, carregam como causadoras do adoecimento de seu filho. Contribuem, também, para percebermos as diferenças dos contextos culturais no enfrentamento das famílias ${ }^{(24)}$.

Um aspecto, porém, que tem sido pouco elucidado é sobre como essas experiências ocorrem ao longo do processo do adoecimento. Sabe-se que o adoecimento varia nos seus 
resultados, alguns são breves e alteram minimamente as atividades habitualmente desenvolvidas; outros são mais estressantes, duram mais tempo em seu curso e podem implicar em perda de habilidades, isolamento do doente; outros podem, ainda, levar à morte. $\mathrm{O}$ adoecimento pode também se caracterizar como uma condição crônica, a qual varia grandemente. Alguns levam a um isolamento devastador do funcionamento que é desejável a um paciente. Outros, enquanto perdem lentamente as habilidades, podem, eventualmente, levar os recursos familiares à exaustão, exigindo institucionalização ${ }^{(29)}$.

Percebe-se o aumento da produção de pesquisas qualitativas que enfocam o caráter multidimensional da relação família e doença mental. Porém ainda são poucas frente à complexidade dessa rede de relações e experiência.

\section{REFERÊNCIAS}

1. Moreno $\mathrm{V}$, Alencastre MB. A trajetória da família do portador de sofrimento psíquico. Rev Esc Enferm USP. 2003;37(2):43-50;

2. Colvero LA, Ide CAC, Rolim MA. Família e doença mental: a difícil convivência com a diferença. Rev Esc Enferm Usp 2004;38(2):197-205.

3. Pejlert A. Being a parent of an adult son or daughter with severe mental illness receiving professional care: parents' narratives. Health Soc Care Commun. 2001;9(4):194-204.

4. Maldonado JG, Urízar AC, Kavanagh DJ. Burden of care and general health in families of patients with schizophrenia. Soc Psychiatry Psychiatr Epidemiol. 2005; 40:899-904.

5. Bandeira M, Calzavara MGP, Varella AAB. Escala de sobrecarga dos familiares de pacientes psiquiátricos: adaptação transcultural para o Brasil (FBIS-BR). J Bras Psiquiatr. 2005;54(3):206-14.

6. Foldemo A, Gullberg M, Ek AC, Bogren L. Quality of life and burden in parents of outpatients with schizophrenia. Soc Psychiatry Psychiatr Epidemiol. 2005;40:133-8.

7. Dyck DG, Short R, Vitaliano PP. Predictors of Burden and infectious illness in schizophrenia caregivers. Psychosomatic Medicine 1999;61:411-9.

8. Rose LE. Families of psychiatric patients: a critical review and future research directions. Archives of Psychiatric Nursing 1996;10(2):67-76.

9. Tannis M, Laidlaw TM, Coverdale JH, Falloon IRH, Kydd RR. Caregivers' stresses when living together or apart from patients with chronic schizophrenia. Community Mental Health Journal 2002;38(4):303-10.

10. Jungbauer J, Wittmund B, Dietrich S, Angermeyer MC. Subjective burden over 12 months in parents of patients with schizophrenia. Arch Psychiatr Nurs. 2003;17(3):126-34.

11. Weiss MG, Cohen A, Eisemberg L. Mental Health. In: Merson $\mathrm{MH}$, Black RE, Mills AJ, editors. International Public Health: diseases, programs, systems, and policies. Gaithersburg (MD): An Aspen Publications; 2001. p. 331-71.

12. World Health Organization. The World Health Report. Mental health: new understanding. New Hope, 2001.

13. Urizar AC, Maldonado JG. Burden of care in families of patients with schizophrenia. Qual Life Res 2006;15:719-24.
14. Koga M, Furegato AR. Convivência com a pessoa esquizofrênica: sobrecarga familiar. Ciência, Cuidado e Saúde 2002; 1(1):75-9.

15. Arthur D. The validity and reliability of the measurement of the concept 'expressed emotion' in the family members and nurses of Hong Kong patients with schizophrenia. Int J Ment Health Nurs 2002;11:192-8.

16. Willetts LE, Leff J. Expressed emotion and schizophrenia: the efficacy of a staff training programme. J Adv Nurs 1997;26:1125-33.

17. Vaughn CE, Leff J. The measurement of expressed emotion in the families of psychiatric patients. Br J Soc Clin Psychol 1976; 15:157-65.

18. Cheng ATA. Expressed emotion: a cross-culturally valid concept? Br J Psychiatry 2002;181:466-7.

19. Bhugra D, McKenzie K. Expressed emotion across cultures. Advances in Psychiatric Treatment 2003;9:342-8.

20. Montagna PLK. Emoções expressas no ambiente familiar e evolução da esquizofrenia [Dissertação]. São Paulo (SP): Faculdade de Medicina, Universidade de São Paulo; 1981.

21. Fukuda, IMK. O convívio com o paciente esquizofrênico: experiência dos familiares [Tese]. São Paulo (SP): Escola de Enfermagem, Universidade de São Paulo; 1989.

22. Jungbauer J, Stelling K, Dietrich S, Angermeyer MC. Schizophrenia: problems of separation in families. J Adv Nurs 2004;47(6): 605-13.

23. Chesla C. Parents' illness models of schizophrenia. Arch Psychiatr Nurs 1989;3(4):218-25.

24. Yamashita M. Family coping with mental illness: a comparative study. Journal of Psychiatric and Mental Health Nursing 1998;5:515-23.

25. Rungreangkulkij S, Chesla C. Smooth a heart with water: Thai mothers care for a child with schizophrenia. Arch of Psychiatr Nurs 2001;15(3):120-7.

26. Mcnab BC, Haslam N, Burnett P. Expressed emotion, attributions, utility beliefs, and distress in parents of young people with first episode psychosis. Psychiatry Research 2007;151:97-106.

27. Hatfleld AB, Spanlol L, Zlpple AM. Expressed Emotion: A family perspective. Schizophr Bull 1987;13(2):221-6.

28. Kleinman A. The illness narratives: suffering, and human conditions. New York: Basic Books; 1988. 